\title{
Spin-3/2 Ising model on a two-layer Bethe lattice with FM/AFM interactions
}

\author{
E.Albayrak, A.Yigit \\ Erciyes University, Department of Physics, 38039, Kayseri, Turkey \\ Received February 2, 2009, in final form May 25, 2009
}

\begin{abstract}
Two-layer Bethe lattice whose lattice sites are occupied with spin-3/2 atoms is solved exactly by using the recursion relations in a pairwise approach for given coordination numbers $q=3,4$ and 6 with equal external magnetic fields acting on the layers. The ferromagnetic (FM) and antiferromagnetic (AFM) interactions for the spins of the upper and lower layers, respectively, and either FM or AFM type interactions between the adjacent spins of the layers are assumed. The phase diagrams of the model are studied on different planes for given system parameters by obtaining the ground state (GS) phase diagrams and the thermal variations of the order parameters and the response functions, i. e. the susceptibility and the specific heat, in detail. It was found that the model presents both second- and first-order phase transitions. The reentrant behavior is seen when the model presents two Néel temperatures for higher $q$ values. The existence of the tricritical point and critical end points is also confirmed.
\end{abstract}

Key words: Bethe lattice, spin, ground state, fm/afm, layer, phase

PACS: $05.50 .+q, 68.35 . R h, 64.60 . C n$

\section{Introduction}

The spin-3/2 Ising model with only bilinear and biquadratic exchange interactions was introduced earlier [1] to explain phase transitions in $\mathrm{DyVO}_{4}$ and its phase diagram was obtained by the use of the mean-field approximation (MFA). Another model was introduced later on to study tricritical properties in ternary fluid mixtures (ethanol-water-carbon-dioxide) that was also solved in the MFA [2]. Since then the spin-3/2 model was studied by many approximate techniques and it was found that its phase diagrams are much richer in comparison with the lower spin systems, i. e. spin- $1 / 2$ and 1 .

In the recent years, in addition to one layer models, the study of magnetic thin films consisting of various magnetic layered structures or superlattices has received intense attention for both theoretical and experimental reasons. Especially the bilayer ones have attracted a great deal of interest with FM/FM, AFM/AFM or FM/AFM type intralayer and either FM or AFM type interlayer interactions. These materials present some interesting novel magnetic properties such as giant magnetoresistance [3], surface magnetic anisotropy [4], enhanced surface magnetic moment [5] and surface magnetoelastic coupling [6], etc. Thus, there is a high potential for technological advances in information storage and retrieval and in synthesis of new magnets for a variety of applications.

Even though the multilayer structures containing spin- $1 / 2$ or spin- 1 have attracted a great deal of interest, the multilayered spin-3/2 models have not received enough attention, i. e. we could only report a few works; the two-layered model with competing interactions was studied by using the interfacial approximation on a square lattice [7], a bilayer Bethe lattice with FM/FM interactions by the use of the recursion relations [8] and on a model with the periodical arrangement of one layer with spin-1/2 and the other layer with spin-3/2 were examined by the use of the effective field theory [9]. We should note that the external magnetic field and bilinear interaction parameter are always in competition for the AFM systems, i. e. the spins in a nearest-neighbor (NN) pair for the AFM system are aligned oppositely but the external magnetic field tries to align them parallelly. 
Thus, due to this competition, one expects to get richer phase diagrams in comparison with the FM/FM case.

Some of the experimental works with FM/AFM interactions may be listed as follows. A positive unidirectional exchange anisotropy was discovered in $\mathrm{AFM}\left(\mathrm{FeF}_{2}\right)$ and $\mathrm{FM}(\mathrm{Fe})$ bilayers cooled through the AFM critical temperature $T_{\mathrm{N}}$ in large magnetic fields [10]. A new route towards enhancing the energy product of permanent magnetic materials, at room temperature, based on FM-AFM exchange interactions has been developed [11]. Molecular beam epitaxy was used in order to grow a $\mathrm{FM} / \mathrm{AFM}\left(\mathrm{Fe} / \mathrm{KCoF}_{3}\right)$ system and the structure and magnetic properties of the exchange biased system were studied experimentally [12]. The synthesized thin film $\mathrm{Co} / \mathrm{CoO}$ was used to study the characteristics of FM/AFM interface such as unidirectional magnetic anisotropy, rotational magnetic hysteresis in relatively high magnetic fields, unusual magnetic aftereffect, etc, [13]. Exchange bias and coercivity enhancement in FM/AFM bilayer, i. e. CoO-Co bilayer [14], the spin configurations in a frustrated FM/AFM thin film system [15], the exchange coupling between FM and AFM layers across a non-magnetic interlayer: $\mathrm{Co} / \mathrm{Cu} / \mathrm{FeMn}$ on $\mathrm{Cu}(001)$ were investigated by magnetic circular dichroism domain imaging [16]. The exchange coupling between AFM NiO and FM films [17] and the magnetization reversal in exchange biased FM/AFM bilayer [18] were investigated.

It should also be noted that exact solutions for realistic systems on regular lattices are generally unavailable. Thus, one has to rely on the approximation methods to obtain, at least, a qualitative picture for the considered system. Thus, a two-layer Bethe lattice for an Ising model was studied by using an iteration technique [19]. The role of interlayer coupling between $\mathrm{CuO}_{2}$ planes on bilayer-group high-Tc superconductors was studied within a simple randomly decorated bilayer Ising model and a Bethe-lattice approach [20]. The behavior of the Ising thin films through the use of layered Bethe lattices and Husimi trees was studied [21]. The phase diagrams of spin-1/2 Ising model on a two-layer Bethe lattice with FM/AFM interactions were studied [22]. Similarly, the phase diagrams of spin-1 Ising model on a two-layer Bethe lattice for the cases of FM/AFM and FM/FM interactions without [23] and with crystal field [24] were studied by using a pairwise approach.

Meanwhile, a Bethe lattice is an infinitely Cayley or regular tree, i. e. a connected graph without circuits, and historically gets its name from the fact that its partition function is exactly that of an Ising model in the Bethe approximation [25]. In this study the original one-layer lattice is replaced with the two-layer Bethe lattice with the same coordination number $q$. It should be mentioned that the one-layer Bethe lattice provides solutions and the results of which are qualitatively better approximations for the regular lattices than the solutions obtained by conventional mean-field theories [26]. We should also point out that the cluster variation method in the pair approximation studies on regular lattices yields the results that are solved exactly for the same model on the Bethe lattice [27]. Of course, the Bethe lattice considerations also have got some limitations, i. e., it predicts a transition temperature which is higher than that for a regular lattice and they are not reliable in predicting the critical exponents [19]. For more discussions about the Bethe lattices, the readers are referred to the [19].

In this work in order to simulate the FM/AFM two-layer Ising model for spin-3/2, two symmetrically placed Bethe lattices with the upper layer having only FM and the lower one having only AFM interactions, each with a branching ratio of $q$ Ising spins, and each of which are coupled via either FM or AFM interactions is considered. In this model we have calculated the partition function exactly and then the model was studied by the use of the recursion relations in a pairwise approach. The name "pairwise approach" is used since we form a pair by taking the adjacent nearest-neighbor $(\mathrm{NN})$ spins from each layer, then one moves from root to the boundary sites (in the thermodynamic limit it goes to infinity) by considering all the interactions at each step. In addition to the GS phase diagrams of the model, the order-parameters, free energy and the response functions are studied to obtain the temperature-dependent phase diagrams for various values of coordination numbers, i. e. $q=3,4$ and 6 .

The remainder of this work is set up as follows. Section 2 is devoted to the introduction of the two-layer Bethe lattice and its Hamiltonian, and to the formulation of the model. The GS 
configurations and its phase diagrams are given in section 3 . The thermal variations of the orderparameters and the response functions are presented in section 4 . The illustrations of the phase diagrams are given in section 5. Finally, a brief summary and conclusions were given in the last section.

\section{Two-layer Bethe lattice and exact formulations}

The spin-3/2 two-layer model is simulated on a Bethe lattice and a pairwise approach is employed for its investigation. In this approach one picks an adjacent NN spin pair from deep inside the two layer lattice, referred to as the central pair, which forms the first-generation spins. This central pair of spins is connected to $q \mathrm{NN}$ spin pairs (i. e. $q$ is the coordination number) which form the second-generation spins. Each pair of spins in the second-generation is joined to $(q-1)$ NN's. Therefore, in total, the second-generation has $q(q-1)$ NN's which form the third-generation and so on to infinity. As a result each spin has $(q+1)$ NN spins, $q$ from its own layer and one from the adjacent layer.

The Ising Hamiltonian of such a two-layer Bethe lattice with an external magnetic field linked to the layers may be given as

$$
\mathcal{H}=-J_{1} \sum_{\langle i j\rangle} S_{i} S_{j}-J_{2} \sum_{\left\langle i^{\prime} j^{\prime}\right\rangle} \sigma_{i^{\prime}} \sigma_{j^{\prime}}-J_{3} \sum_{\left\langle i i^{\prime}\right\rangle} S_{i} \sigma_{i^{\prime}}-H_{1} \sum_{i} S_{i}-H_{2} \sum_{i^{\prime}} \sigma_{i^{\prime}},
$$

where $S_{i}$ and $\sigma_{i^{\prime}}$ are spin-3/2 with the values $\pm 3 / 2$ and $\pm 1 / 2$ and refer to the spins in the upper, $G_{1}$, and lower, $G_{2}$, layers, respectively. $J_{1}>0$ and $J_{2}<0$ are the intralayer bilinear interactions of the NN spins of the layers. Thus, the first and second summations are over all NN sites of $G_{1}$ and $G_{2}$, respectively. $J_{3}$ is the interlayer bilinear interaction of adjacent NN spins between the layers. Therefore, the third summation runs over all the adjacent neighboring sites of $G_{1}$ and $G_{2}$. The layers are assumed to be under the effect of external magnetic fields $H_{1}$ and $H_{2}$. Therefore, the fourth and final sums run over all the lattice sites in each layer, respectively. Let us come to the formulation with the partition function which is given by the definition as

$$
\mathbb{Z}=\sum_{\text {All config. }} \mathrm{e}^{-\beta \mathcal{H}}=\sum_{\mathrm{Spc}} P(\mathrm{Spc})
$$

where $P(\mathrm{Spc})$ is the unnormalized probability distribution, see [28] for its detailed presentation, $\beta=1 / k T$ is the inverse temperature and $k$ is the Boltzmann constant, and the summation runs over all the spin configurations (Spc). Starting from the central pair of spins on the Bethe lattice made up of $q$ separate branches connecting each pair of spins, one follows only one of the branches of the tree out of $q$. Therefore, for a full formulation we have to define the partition function for each of these separate branches referred to as $g_{n}(S, \sigma)$. It should be mentioned that each spin $S_{i}$ and spin $\sigma_{i^{\prime}}$ with spin-3/2 can take the values $\pm 3 / 2$ and $\pm 1 / 2$. Thus, we have to define sixteen $g_{n}(S, \sigma)$ functions for $4^{2}=16$ configurations for each pair of spins. As a result, we have obtained 15 functions as the ratios of these partition functions of the separate branches on the two layer Bethe lattice as follows:

$$
\begin{aligned}
& A_{n}=\frac{g_{n}\left(\frac{3}{2}, \frac{3}{2}\right)}{g_{n}\left(-\frac{1}{2},-\frac{1}{2}\right)}, \quad B_{n}=\frac{g_{n}\left(\frac{3}{2},-\frac{3}{2}\right)}{g_{n}\left(-\frac{1}{2},-\frac{1}{2}\right)}, \quad C_{n}=\frac{g_{n}\left(\frac{3}{2}, \frac{1}{2}\right)}{g_{n}\left(-\frac{1}{2},-\frac{1}{2}\right)}, \\
& D_{n}=\frac{g_{n}\left(\frac{3}{2},-\frac{1}{2}\right)}{g_{n}\left(-\frac{1}{2},-\frac{1}{2}\right)}, \quad E_{n}=\frac{g_{n}\left(-\frac{3}{2}, \frac{3}{2}\right)}{g_{n}\left(-\frac{1}{2},-\frac{1}{2}\right)}, \quad F_{n}=\frac{g_{n}\left(-\frac{3}{2},-\frac{3}{2}\right)}{g_{n}\left(-\frac{1}{2},-\frac{1}{2}\right)}, \\
& G_{n}=\frac{g_{n}\left(-\frac{3}{2}, \frac{1}{2}\right)}{g_{n}\left(-\frac{1}{2},-\frac{1}{2}\right)}, \quad H_{n}=\frac{g_{n}\left(-\frac{3}{2},-\frac{1}{2}\right)}{g_{n}\left(-\frac{1}{2},-\frac{1}{2}\right)}, \quad I_{n}=\frac{g_{n}\left(\frac{1}{2}, \frac{3}{2}\right)}{g_{n}\left(-\frac{1}{2},-\frac{1}{2}\right)}, \\
& J_{n}=\frac{g_{n}\left(\frac{1}{2},-\frac{3}{2}\right)}{g_{n}\left(-\frac{1}{2},-\frac{1}{2}\right)}, \quad K_{n}=\frac{g_{n}\left(\frac{1}{2}, \frac{1}{2}\right)}{g_{n}\left(-\frac{1}{2},-\frac{1}{2}\right)}, \quad L_{n}=\frac{g_{n}\left(\frac{1}{2},-\frac{1}{2}\right)}{g_{n}\left(-\frac{1}{2},-\frac{1}{2}\right)}, \\
& M_{n}=\frac{g_{n}\left(-\frac{1}{2}, \frac{3}{2}\right)}{g_{n}\left(-\frac{1}{2},-\frac{1}{2}\right)}, \quad N_{n}=\frac{g_{n}\left(-\frac{1}{2},-\frac{3}{2}\right)}{g_{n}\left(-\frac{1}{2},-\frac{1}{2}\right)}, \quad R_{n}=\frac{g_{n}\left(-\frac{1}{2}, \frac{1}{2}\right)}{g_{n}\left(-\frac{1}{2},-\frac{1}{2}\right)} \text {. }
\end{aligned}
$$


They are related to each other as follows:

$$
\Delta_{n}=f\left(A_{n-1}, B_{n-1}, \ldots, N_{n-1}, R_{n-1}\right),
$$

where $\Delta$ refer to the 15 functions given in equation (3). Thus, each of these functions with $n$ (number of the shells from root to the boundary sites) is a function of itself and the other functions with $(n-1)$. Therefore, they are totally nonlinear and so they form recursion relations, the explicit expressions of which are too long to be given in here. Their numerical values, therefore, can easily be obtained by using an iteration method for the given system parameters. Meanwhile, the choice of the recursion relations, i. e. the ratios of these $g_{n}(S, \sigma)$ functions, are completely arbitrary, i.e, this choice does not change the behavior of the system.

These recursion relations may not have any physical meaning, but they do reflect the critical behavior of the system as it will be obvious from the behavior of the order-parameters. One can easily express the magnetizations and other thermodynamic quantities in terms of these exact recursion relations. Thus, we can say that in the thermodynamic limit, i. e. $n \rightarrow \infty$, these recursion relations determine the states of the system, and they may be refereed to as equations of state.

Magnetizations $M_{1}$ and $M_{2}$ of the layers $G_{1}$ and $G_{2}$, respectively, are the order-parameters and are defined as follows:

$$
M_{1}=\langle S\rangle \text { and } M_{2}=\langle\sigma\rangle,
$$

where $\langle\ldots\rangle$ denotes the usual thermal average. Magnetizations $M_{1}$ and $M_{2}$ are calculated in terms of recursion relations as follows:

$$
\begin{aligned}
M_{1}= & \left\{3 \left[\mathrm{e}^{\beta\left(\frac{9}{4} J_{3}+\frac{3}{2} H_{1}+\frac{3}{2} H_{2}\right)} A_{n}^{q}+\mathrm{e}^{\beta\left(-\frac{9}{4} J_{3}+\frac{3}{2} H_{1}-\frac{3}{2} H_{2}\right)} B_{n}^{q}+\mathrm{e}^{\beta\left(\frac{3}{4} J_{3}+\frac{3}{2} H_{1}+\frac{1}{2} H_{2}\right)} C_{n}^{q}\right.\right. \\
& +\mathrm{e}^{\beta\left(-\frac{3}{4} J_{3}+\frac{3}{2} H_{1}-\frac{1}{2} H_{2}\right)} D_{n}^{q}-\mathrm{e}^{\beta\left(-\frac{9}{4} J_{3}-\frac{3}{2} H_{1}+\frac{3}{2} H_{2}\right)} E_{n}^{q}-\mathrm{e}^{\beta\left(\frac{9}{4} J_{3}-\frac{3}{2} H_{1}-\frac{3}{2} H_{2}\right)} F_{n}^{q} \\
& \left.-\mathrm{e}^{\beta\left(-\frac{3}{4} J_{3}-\frac{3}{2} H_{1}+\frac{1}{2} H_{2}\right)} G_{n}^{q}-\mathrm{e}^{\beta\left(\frac{3}{4} J_{3}-\frac{3}{2} H_{1}-\frac{1}{2} H_{2}\right)} H_{n}^{q}\right]+\left[\mathrm{e}^{\beta\left(\frac{3}{4} J_{3}+\frac{1}{2} H_{1}+\frac{3}{2} H_{2}\right)} I_{n}^{q}\right. \\
& +\mathrm{e}^{\beta\left(-\frac{3}{4} J_{3}+\frac{1}{2} H_{1}-\frac{3}{2} H_{2}\right)} J_{n}^{q}+\mathrm{e}^{\beta\left(\frac{1}{4} J_{3}+\frac{1}{2} H_{1}+\frac{1}{2} H_{2}\right)} K_{n}^{q}+\mathrm{e}^{\beta\left(-\frac{1}{4} J_{3}+\frac{1}{2} H_{1}-\frac{1}{2} H_{2}\right)} L_{n}^{q} \\
& -\mathrm{e}^{\beta\left(-\frac{3}{4} J_{3}-\frac{1}{2} H_{1}+\frac{3}{2} H_{2}\right)} M_{n}^{q}-\mathrm{e}^{\beta\left(\frac{3}{4} J_{3}-\frac{1}{2} H_{1}-\frac{3}{2} H_{2}\right)} N_{n}^{q}-\mathrm{e}^{\beta\left(-\frac{1}{4} J_{3}-\frac{1}{2} H_{1}+\frac{1}{2} H_{2}\right)} R_{n}^{q} \\
& \left.\left.-\mathrm{e}^{\beta\left(\frac{1}{4} J_{3}-\frac{1}{2} H_{1}-\frac{1}{2} H_{2}\right)}\right]\right\} / 2 \mathbb{Z}
\end{aligned}
$$

and

$$
\begin{aligned}
M_{2}= & \left\{3 \left[\mathrm{e}^{\beta\left(\frac{9}{4} J_{3}+\frac{3}{2} H_{1}+\frac{3}{2} H_{2}\right)} A_{n}^{q}-\mathrm{e}^{\beta\left(-\frac{9}{4} J_{3}+\frac{3}{2} H_{1}-\frac{3}{2} H_{2}\right)} B_{n}^{q}+\mathrm{e}^{\beta\left(-\frac{9}{4} J_{3}-\frac{3}{2} H_{1}+\frac{3}{2} H_{2}\right)} E_{n}^{q}\right.\right. \\
& -\mathrm{e}^{\beta\left(\frac{9}{4} J_{3}-\frac{3}{2} H_{1}-\frac{3}{2} H_{2}\right)} F_{n}^{q}+\mathrm{e}^{\beta\left(\frac{3}{4} J_{3}+\frac{1}{2} H_{1}+\frac{3}{2} H_{2}\right)} I_{n}^{q}-\mathrm{e}^{\beta\left(-\frac{3}{4} J_{3}+\frac{1}{2} H_{1}-\frac{3}{2} H_{2}\right)} J_{n}^{q} \\
& \left.+\mathrm{e}^{\beta\left(-\frac{3}{4} J_{3}-\frac{1}{2} H_{1}+\frac{3}{2} H_{2}\right)} M_{n}^{q}-\mathrm{e}^{\beta\left(\frac{3}{4} J_{3}-\frac{1}{2} H_{1}-\frac{3}{2} H_{2}\right)} N_{n}^{q}\right]+\left[\mathrm{e}^{\beta\left(\frac{3}{4} J_{3}+\frac{3}{2} H_{1}+\frac{1}{2} H_{2}\right)} C_{n}^{q}\right. \\
& -\mathrm{e}^{\beta\left(-\frac{3}{4} J_{3}+\frac{3}{2} H_{1}-\frac{1}{2} H_{2}\right)} D_{n}^{q}+\mathrm{e}^{\beta\left(-\frac{3}{4} J_{3}-\frac{3}{2} H_{1}+\frac{1}{2} H_{2}\right)} G_{n}^{q}-\mathrm{e}^{\beta\left(\frac{3}{4} J_{3}-\frac{3}{2} H_{1}-\frac{1}{2} H_{2}\right)} H_{n}^{q} \\
& +\mathrm{e}^{\beta\left(\frac{1}{4} J_{3}+\frac{1}{2} H_{1}+\frac{1}{2} H_{2}\right)} K_{n}^{q}-\mathrm{e}^{\beta\left(-\frac{1}{4} J_{3}+\frac{1}{2} H_{1}-\frac{1}{2} H_{2}\right)} L_{n}^{q}+\mathrm{e}^{\beta\left(-\frac{1}{4} J_{3}-\frac{1}{2} H_{1}+\frac{1}{2} H_{2}\right)} R_{n}^{q} \\
& \left.\left.-\mathrm{e}^{\beta\left(\frac{1}{4} J_{3}-\frac{1}{2} H_{1}-\frac{1}{2} H_{2}\right)}\right]\right\} / 2 \mathbb{Z}
\end{aligned}
$$

where

$$
\begin{aligned}
\mathbb{Z}= & \mathrm{e}^{\beta\left(\frac{9}{4} J_{3}+\frac{3}{2} H_{1}+\frac{3}{2} H_{2}\right)} A_{n}^{q}+\mathrm{e}^{\beta\left(-\frac{9}{4} J_{3}+\frac{3}{2} H_{1}-\frac{3}{2} H_{2}\right)} B_{n}^{q} \\
& +\mathrm{e}^{\beta\left(\frac{3}{4} J_{3}+\frac{3}{2} H_{1}+\frac{1}{2} H_{2}\right)} C_{n}^{q}+\mathrm{e}^{\beta\left(-\frac{3}{4} J_{3}+\frac{3}{2} H_{1}-\frac{1}{2} H_{2}\right)} D_{n}^{q} \\
& +\mathrm{e}^{\beta\left(-\frac{9}{4} J_{3}-\frac{3}{2} H_{1}+\frac{3}{2} H_{2}\right)} E_{n}^{q}+\mathrm{e}^{\beta\left(\frac{9}{4} J_{3}-\frac{3}{2} H_{1}-\frac{3}{2} H_{2}\right)} F_{n}^{q} \\
& +\mathrm{e}^{\beta\left(-\frac{3}{4} J_{3}-\frac{3}{2} H_{1}+\frac{1}{2} H_{2}\right)} G_{n}^{q}+\mathrm{e}^{\beta\left(\frac{3}{4} J_{3}-\frac{3}{2} H_{1}-\frac{1}{2} H_{2}\right)} H_{n}^{q} \\
& +\mathrm{e}^{\beta\left(\frac{3}{4} J_{3}+\frac{1}{2} H_{1}+\frac{3}{2} H_{2}\right)} I_{n}^{q}+\mathrm{e}^{\beta\left(-\frac{3}{4} J_{3}+\frac{1}{2} H_{1}-\frac{3}{2} H_{2}\right)} J_{n}^{q}
\end{aligned}
$$




$$
\begin{aligned}
& +\mathrm{e}^{\beta\left(\frac{1}{4} J_{3}+\frac{1}{2} H_{1}+\frac{1}{2} H_{2}\right)} K_{n}^{q}+\mathrm{e}^{\beta\left(-\frac{1}{4} J_{3}+\frac{1}{2} H_{1}-\frac{1}{2} H_{2}\right)} L_{n}^{q} \\
& +\mathrm{e}^{\beta\left(-\frac{3}{4} J_{3}-\frac{1}{2} H_{1}+\frac{3}{2} H_{2}\right)} M_{n}^{q}+\mathrm{e}^{\beta\left(+\frac{3}{4} J_{3}-\frac{1}{2} H_{1}-\frac{3}{2} H_{2}\right)} N_{n}^{q} \\
& +\mathrm{e}^{\beta\left(-\frac{1}{4} J_{3}-\frac{1}{2} H_{1}+\frac{1}{2} H_{2}\right)} R_{n}^{q}+\mathrm{e}^{\beta\left(+\frac{1}{4} J_{3}-\frac{1}{2} H_{1}-\frac{1}{2} H_{2}\right)} .
\end{aligned}
$$

The two response functions are susceptibility and specific heat. Thus, the constant field susceptibilities of the layers $G_{1}$ and $G_{2}$ are given by the definitions as follows:

$$
\chi_{1 \mathrm{~T}}=\lim _{H_{1} \rightarrow H} \frac{\partial M_{1}}{\partial H_{1}} \text { and } \chi_{2 \mathrm{~T}}=\lim _{H_{2} \rightarrow H} \frac{\partial M_{2}}{\partial H_{2}}
$$

and the total susceptibility of the two-layer is just the sum of the susceptibilities

$$
\chi_{T}=\chi_{1 \mathrm{~T}}+\chi_{2 \mathrm{~T}} .
$$

In order to obtain the specific heat, the free energy in terms of recursion relations is needed. Therefore, using the definition of the free energy $F=-k T \log \mathbb{Z}$ and equations (3) and (8) in the thermodynamic limit as $n \rightarrow \infty$, one can obtain an exact expression for the free energy in terms of recursion relations. Thus, after some straightforward mathematical calculations there was found the following:

$$
-\beta F=\frac{q}{2} \log \mathbb{W}+\frac{2-q}{2} \log \mathbb{Z}
$$

where

$$
\begin{aligned}
\mathbb{W}= & \mathrm{e}^{\beta\left(-\frac{3}{4} J_{1}-\frac{3}{4} J_{2}+\frac{9}{4} J_{3}+\frac{3}{2} H_{1}+\frac{3}{2} H_{2}\right)} A_{n}^{q-1}+\mathrm{e}^{\beta\left(-\frac{3}{4} J_{1}+\frac{3}{4} J_{2}-\frac{9}{4} J_{3}+\frac{3}{2} H_{1}-\frac{3}{2} H_{2}\right)} B_{n}^{q-1} \\
& +\mathrm{e}^{\beta\left(-\frac{3}{4} J_{1}-\frac{1}{4} J_{2}+\frac{3}{4} J_{3}+\frac{3}{2} H_{1}+\frac{1}{2} H_{2}\right)} C_{n}^{q-1}+\mathrm{e}^{\beta\left(-\frac{3}{4} J_{1}+\frac{1}{4} J_{2}-\frac{3}{4} J_{3}+\frac{3}{2} H_{1}-\frac{1}{2} H_{2}\right)} D_{n}^{q-1} \\
& +\mathrm{e}^{\beta\left(\frac{3}{4} J_{1}-\frac{3}{4} J_{2}-\frac{9}{4} J_{3}-\frac{3}{2} H_{1}+\frac{3}{2} H_{2}\right)} E_{n}^{q-1}+\mathrm{e}^{\beta\left(\frac{3}{4} J_{1}+\frac{3}{4} J_{2}+\frac{9}{4} J_{3}-\frac{3}{2} H_{1}-\frac{3}{2} H_{2}\right)} F_{n}^{q-1} \\
& +\mathrm{e}^{\beta\left(\frac{3}{4} J_{1}-\frac{1}{4} J_{2}-\frac{3}{4} J_{3}-\frac{3}{2} H_{1}+\frac{1}{2} H_{2}\right)} G_{n}^{q-1}+\mathrm{e}^{\beta\left(\frac{3}{4} J_{1}+\frac{1}{4} J_{2}+\frac{3}{4} J_{3}-\frac{3}{2} H_{1}-\frac{1}{2} H_{2}\right)} H_{n}^{q-1} \\
& +\mathrm{e}^{\beta\left(-\frac{1}{4} J_{1}-\frac{3}{4} J_{2}+\frac{3}{4} J_{3}+\frac{1}{2} H_{1}+\frac{3}{2} H_{2}\right)} I_{n}^{q-1}+\mathrm{e}^{\beta\left(-\frac{1}{4} J_{1}+\frac{3}{4} J_{2}-\frac{3}{4} J_{3}+\frac{1}{2} H_{1}-\frac{3}{2} H_{2}\right)} J_{n}^{q-1} \\
& +\mathrm{e}^{\beta\left(-\frac{1}{4} J_{1}-\frac{1}{4} J_{2}+\frac{1}{4} J_{3}+\frac{1}{2} H_{1}+\frac{1}{2} H_{2}\right)} K_{n}^{q-1}+\mathrm{e}^{\beta\left(-\frac{1}{4} J_{1}+\frac{1}{4} J_{2}-\frac{1}{4} J_{3}+\frac{1}{2} H_{1}-\frac{1}{2} H_{2}\right)} L_{n}^{q-1} \\
& +\mathrm{e}^{\beta\left(\frac{1}{4} J_{1}-\frac{3}{4} J_{2}-\frac{3}{4} J_{3}-\frac{1}{2} H_{1}+\frac{3}{2} H_{2}\right)} M_{n}^{q-1}+\mathrm{e}^{\beta\left(\frac{1}{4} J_{1}+\frac{3}{4} J_{2}+\frac{3}{4} J_{3}-\frac{1}{2} H_{1}-\frac{3}{2} H_{2}\right)} N_{n}^{q-1} \\
& +\mathrm{e}^{\beta\left(\frac{1}{4} J_{1}-\frac{1}{4} J_{2}-\frac{1}{4} J_{3}-\frac{1}{2} H_{1}+\frac{1}{2} H_{2}\right)} R_{n}^{q-1}+\mathrm{e}^{\beta\left(\frac{1}{4} J_{1}+\frac{1}{4} J_{2}+\frac{1}{4} J_{3}-\frac{1}{2} H_{1}-\frac{1}{2} H_{2}\right)}
\end{aligned}
$$

which is used to find the places of the first-order phase transition temperatures, if any exist, and the stable solutions of the model. As a result, the specific heat at a constant magnetic field is defined as

$$
C_{H}=T\left(\frac{\partial S}{\partial T}\right)_{H}=-T\left(\frac{\partial^{2} F}{\partial T^{2}}\right)_{H},
$$

where $H$ refers to either $H_{1}$ or $H_{2}$, and $S$ is the entropy and it may be given in a more suitable form as

$$
C / k=-\beta^{\prime}\left[2 \beta^{\prime} \frac{\partial\left(F / J_{i}\right)}{\partial \beta^{\prime}}+\beta^{\prime 2} \frac{\partial^{2}\left(F / J_{i}\right)}{\partial \beta^{\prime 2}}\right],
$$

where $i=1$ or 2 , i. e. $J_{i}$ refers to intralayer interaction of the upper or lower layer, respectively, and which is not as simple as it is seen, since it includes the first- and second-partial derivatives of the recursion relations with respect to $\beta^{\prime}=\beta J_{i}$. Therefore, the explicit equation is too long to be given here.

So far we have obtained the formulations for general interactions only, but for the lower layer $G_{2}$ we have assumed AFM interactions. Therefore, we have to divide the layer $G_{2}$ into sublattices A 
and B. But for the sake of the formulations, we have to partition the upper layer $G_{1}$ into sublattices $\mathrm{A}$ and $\mathrm{B}$ too in a pairwise approach. Hence, the recursion relations for each layer in terms of the sublattices may be written as follows:

$$
\left\{A_{n}, B_{n}, \ldots, N_{n}, R_{n}\right\} \rightarrow \begin{aligned}
& \left\{A_{n}^{A}, B_{n}^{A}, \ldots, N_{n}^{A}, R_{n}^{A}\right\} \\
& \left\{A_{n}^{B}, B_{n}^{B}, \ldots, N_{n}^{B}, R_{n}^{B}\right\}
\end{aligned} \quad \text { "for even } n ",
$$

The notable point of this approach is that non-staggered phases are described by the single fixed points $\left\{A_{n}, B_{n}, \ldots, N_{n}, R_{n}\right\} \rightarrow\{A, B, \ldots, N, R\}$, while the staggered phases appear as two-cycle double points as indicated above.

In addition, the sublattice magnetizations for the layers $G_{1}$ and $G_{2}$, since each spin only interacts with its NN's from its own layer and a NN from the adjacent layer with the same sublattice, i. e. A or B, could be written as

$$
\left\{M_{1,2}\right\} \rightarrow \begin{aligned}
& \left\{M_{1 \mathrm{~A}}, M_{2 \mathrm{~A}}\right\} \\
& \left\{M_{1 \mathrm{~B}}, M_{2 \mathrm{~B}}\right\}
\end{aligned} \quad \text { "for even } n "
$$

The free energies of the sublattices are of the form

$$
\left\{F\left(A_{n}, \ldots, R_{n}\right)\right\} \rightarrow \begin{aligned}
& \left\{F\left(A_{n}^{A}, \ldots, R_{n}^{A}\right)\right\} \quad \text { "for even } n " \\
& \left\{F\left(A_{n}^{B}, \ldots, R_{n}^{B}\right)\right\}
\end{aligned} \text { "for odd } n ",
$$

and then the specific heat is to be defined accordingly, that is

$$
\left\{C\left(A_{n}, \ldots, R_{n}\right)\right\} \rightarrow \begin{aligned}
& \left\{C\left(A_{n}^{A}, \ldots, R_{n}^{A}\right)\right\} \quad \text { "for even } n " \\
& \left\{C\left(A_{n}^{B}, \ldots, R_{n}^{B}\right)\right\}
\end{aligned} \text { "for odd } n ",
$$

and finally the susceptibility is given as

$$
\left\{\chi\left(A_{n}, \ldots, R_{n}\right)\right\} \rightarrow \begin{aligned}
& \left\{\chi_{1}\left(A_{n}^{A}, \ldots, R_{n}^{A}\right)+\chi_{2}\left(A_{n}^{A}, \ldots, R_{n}^{A}\right)\right\} \\
& \left\{\chi_{1}\left(A_{n}^{B}, \ldots, R_{n}^{B}\right)+\chi_{2}\left(A_{n}^{B}, \ldots, R_{n}^{B}\right)\right\}
\end{aligned} \quad \text { "for even } n ",
$$

The next section is devoted to the obtaining of the GS phase diagrams and to the thermal variations of the order-parameters and response functions.

\section{The ground state phase diagrams}

In this section, the GS phase diagrams are calculated in order to determine the stable solutions of the model together with the free energy. Although the GS phase diagrams can only help us at zero temperature, the zero temperature configurations are very important as a starting point in obtaining the temperature dependent phase diagrams.

With the assumption that the layers are linked with equal external magnetic field, i. e. $H=$ $H_{1}=H_{2}$, the GS phase diagrams are obtained on the $\left(J_{i} /\left|J_{j}\right|, J_{3} / q\left|J_{j}\right|\right)$ planes for the given values of $H / q\left|J_{j}\right|$ and on the $\left(H / q\left|J_{j}\right|, J_{3} / q\left|J_{j}\right|\right)$ planes for the given $J_{i} /\left|J_{j}\right|$ values with $i, j=1$ or 2 and $i \neq j$. The GS energies are obtained from equation (1) in units of $\left|J_{k}\right|$ by rewriting it as:

$$
\frac{E}{q\left|J_{k}\right|}=-\sum_{\langle\text {plaq }\rangle}\left[\frac{J_{1}}{\left|J_{k}\right|} S_{i} S_{j}+\frac{J_{2}}{\left|J_{k}\right|} \sigma_{i^{\prime}} \sigma_{j^{\prime}}+\frac{J_{3}}{q\left|J_{k}\right|}\left(S_{i} \sigma_{i^{\prime}}+S_{j} \sigma_{j^{\prime}}\right)+\frac{H}{q\left|J_{k}\right|}\left(S_{i}+S_{j}+\sigma_{i^{\prime}}+\sigma_{j^{\prime}}\right)\right],
$$

where $k=1$ or 2 and the summation is over all the central plaquette which consists of four NN pairs of the two-layer system with one pair $\langle i j\rangle$ from layer $G_{1}$, one pair $\left\langle i^{\prime} j^{\prime}\right\rangle$ from layer $G_{2}$, and two pairs $\left\langle i i^{\prime}\right\rangle$ and $\left\langle j j^{\prime}\right\rangle$ connecting layers $G_{1}$ and $G_{2}$ between the adjacent spins only. The GS phase diagrams are calculated by comparing the values of the energy for different spin configurations and then the GS configurations are the ones with the lowest energy. The coordination number $q$ 


\begin{tabular}{|c|cc|}
\hline I & $+3 / 2$ & $+3 / 2$ \\
& $+3 / 2$ & $+3 / 2$ \\
\hline \multirow{2}{*}{ IV } & $\pm 3 / 2$ & $\pm 3 / 2$ \\
& $\mp 3 / 2$ & $\mp 3 / 2$ \\
\hline
\end{tabular}

\begin{tabular}{|c|cc|}
\hline \multirow{2}{*}{ II } & $\pm 3 / 2$ & $\mp 3 / 2$ \\
& $\mp 3 / 2$ & $\pm 3 / 2$ \\
\hline \multirow{2}{*}{$\mathbf{V}$} & $\mp 3 / 2$ & $\pm 3 / 2$ \\
& $\mp 3 / 2$ & $\pm 3 / 2$ \\
\hline
\end{tabular}

\begin{tabular}{|l|ll|}
\hline \multirow{2}{*}{ III } & $+3 / 2$ & $+3 / 2$ \\
& $\mp 3 / 2$ & $\pm 3 / 2$ \\
\hline
\end{tabular}

is hidden in the GS energy equations. Thus, they are obtained for general $q$. We only consider the case with positive values of $H / q\left|J_{k}\right|$, then there is a preferred direction in space. For negative values of $H / q\left|J_{k}\right|$ the magnetizations only change the sign due to the symmetry requirements, $M(-H)=-M(H)$. Thus, the following five different types of GS configurations are found for the central plaquette deep inside the two-layer Bethe lattice as: While phase I is the FM phase, phase II is the AFM phase. Phase III is the surface FM phase, i. e. layers $G_{1}$ and $G_{2}$ are FM and AFM types, respectively. Phase IV is a compensated phase with the layers being FM type but the interactions between them is AFM type. The last one, phase $\mathrm{V}$, is a mixed phase with AFM intralayer interactions and with FM interlayer interactions. Thus, these last two phases are exactly opposite to each other.

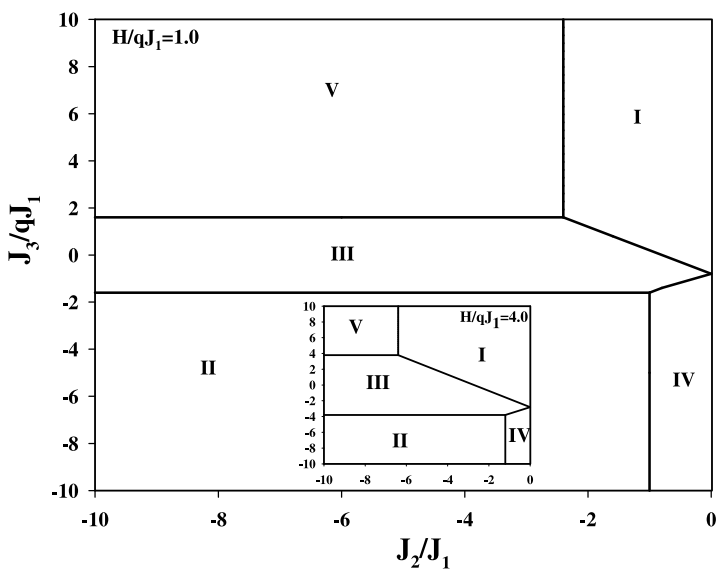

(a)

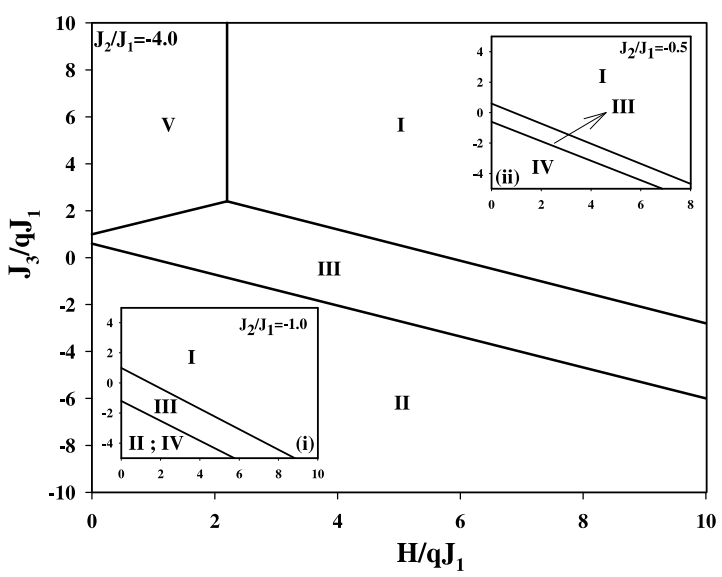

(c)

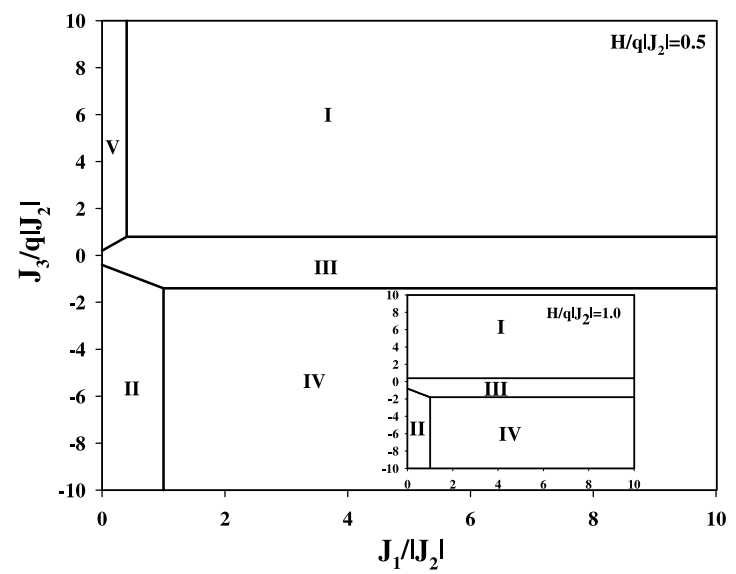

(b)

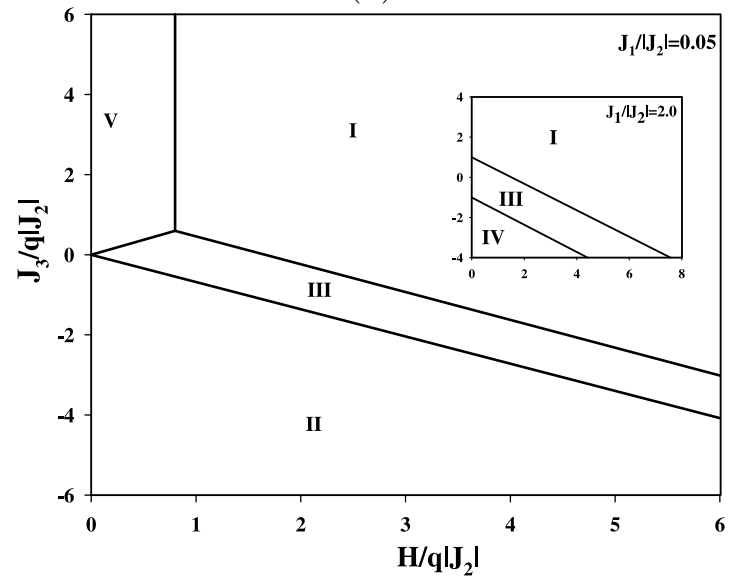

(d)

Figure 1. The GS phase diagrams of the bilayer FM-AFM spin- $3 / 2$ Ising model; (a) on the $\left(J_{2} / J_{1}, J_{3} / q J_{1}\right)$ planes for $H / q J_{1}=1.0$ and in the inset for $H / q J_{1}=4.0$, (b) on the $\left(J_{1} /\left|J_{2}\right|, J_{3} / q\left|J_{2}\right|\right)$ planes for $H / q\left|J_{2}\right|=0.5$ and in the inset for $H / q\left|J_{2}\right|=1.0$, (c) on the $\left(H / q J_{1}, J_{3} / q J_{1}\right)$ planes for $J_{2} / J_{1}=-4.0$ and in the insets $(i)$ for $J_{2} / J_{1}=-1.0$ and $(i i)$ for $J_{2} / J_{1}=-0.5$ and (d) on the $\left(H / q\left|J_{2}\right|, J_{3} / q\left|J_{2}\right|\right)$ planes for $J_{1} /\left|J_{2}\right|=0.05$ and in the inset for $J_{1} /\left|J_{2}\right|=2.0$. 
The GS phase diagrams are studied for the cases with $J_{1}>0, J_{2}<0, H>0$ and either $J_{3}>0$ or $J_{3}<0$. The first GS phase diagrams are obtained on the $\left(J_{2} / J_{1}, J_{3} / q J_{1}\right)$ plane for $H / q J_{1}=1.0$ and in the inset for $H / q J_{1}=4.0$, see figure 1a. It is clear that all five GS configurations exist and, as $H / q J_{1}$ increases the regions covered by the phases, containing the FM configurations, increase. The next two GS phase diagrams, figure $1 \mathrm{~b}$, are obtained on the $\left(J_{1} /\left|J_{2}\right|, J_{3} / q\left|J_{2}\right|\right)$ planes for $H / q\left|J_{2}\right|=0.5$ and in the inset for 1.0. Only phase I is seen in the upper half plane, the phases II, III and IV are contained in the lower half for $H / q\left|J_{2}\right|=1.0$. But for $H / q\left|J_{2}\right|=0.5$, phase $V$ is also seen at lower values of $J_{1} /\left|J_{2}\right|$ in the upper half plane. And the next two are obtained by changing the roles of $J_{2} / J_{1}$ and $H / q J_{1}$ in comparison with the figure 1 a and presented in figure $1 \mathrm{c}$. They are obtained for $J_{2} / J_{1}=-4.0$ and in the insets $(i)$ and $(i i)$ for $J_{2} / J_{1}=-1.0$ and -0.5 , respectively. We see that the phases II and IV exist in the same region and which are separated by a diagonal multiphase line from phase III and which is separated from phase I with another multiphase line parallel to the first one as seen in the inset $(i)$. In the inset $(i i)$ for $J_{2} / J_{1}=-0.5$, the phases II and IV do not coexist anymore. Instead, only phase IV is seen. The opposite occurs in the main figure for $J_{2} / J_{1}=-4.0$, i. e. now we see phase II instead of phase IV. The phase $\mathrm{V}$ also appears in the main figure in comparison with the insets. The last two are illustrated in figure $1 \mathrm{~d}$ and are obtained on the $\left(H / q\left|J_{2}\right|, J_{3} / q\left|J_{2}\right|\right)$ planes for $J_{1} /\left|J_{2}\right|=0.05$ and in the inset for 2.0. Again the roles of $J_{1} /\left|J_{2}\right|$ and $H / q\left|J_{2}\right|$ are exchanged in comparison with figure $1 \mathrm{~b}$. The phases I, II, III and V are seen in the main figure, but for $J_{1} /\left|J_{2}\right|=2.0$, phase $\mathrm{V}$ has disappeared and phase II is replaced by phase IV.

These are all the distinct types of the GS phase diagrams of the spin-3/2 Ising model and which are to be used as our guide in obtaining the temperature dependent phase diagrams. Our next step is to analyse the order-parameters and the response functions, since the nature of the phase transitions are determined from their thermal behavior.

\section{Thermal variations of the order-parameters and response functions}

Now we study the temperature variations of the order-parameters and the response functions to determine the types of the phase transitions. The existence and the behavior of the second-order phase transition temperatures, $T_{\mathrm{N}}$, i. e. Néel temperatures, and the first-order phase transition temperatures, $T_{\mathrm{t}}$, are studied in different phase regions. While the thermal variations of the sublattice magnetizations of layers are given as main figures, the thermal variations of the response functions are given as insets. We only illustrate the case with $q=6$ due to the similarities with the cases for $q=3$ and 4 .

Thermal variations of the order-parameters and response functions of phase II, the AFM phase, are illustrated in figure $2 \mathrm{a}$. As seen $M_{1 \mathrm{~A}}=M_{2 \mathrm{~B}}=1.5$ and $M_{2 \mathrm{~A}}=M_{1 \mathrm{~B}}=-1.5$ at zero temperature as expected, but as the temperature increases they split very slowly. The sublattice magnetizations decrease as the temperature increases. Then, at the $T_{\mathrm{N}}, M_{1 \mathrm{~A}}$ and $M_{2 \mathrm{~A}}$ combine with $M_{1 \mathrm{~B}}$ and $M_{2 \mathrm{~B}}$ at different values of the layer magnetizations, respectively. The total susceptibilities and the specific heat present peaks at the $T_{\mathrm{N}}$ as expected. Figure $2 \mathrm{~b}$ shows the outcome of varying the temperature for phase III. As expected $M_{1 \mathrm{~A}}=M_{1 \mathrm{~B}}$ and $M_{2 \mathrm{~B}}=-M_{2 \mathrm{~A}}$ at zero temperature and as the temperature increases $M_{1 \mathrm{~A}}$ and $M_{1 \mathrm{~B}}$ separate slowly and as $k T / J_{1}$ increases further, they combine at the $T_{\mathrm{N}}$. Similarly, $M_{2 \mathrm{~A}}$ and $M_{2 \mathrm{~B}}$ decrease from above and below with the increase of $k T / J_{1}$ and they also combine at the same $T_{\mathrm{N}}$ as $M_{1 \mathrm{~A}}$ and $M_{1 \mathrm{~B}}$. Again, the response functions show that the peaks are located at the $T_{\mathrm{N}}$. Phase IV is the compensated phase with a big competition between $H$ and $J_{3}$, since $H$ and $J_{3}<0$ favor FM and AFM phases, respectively. $M_{2 \mathrm{~A}}=M_{2 \mathrm{~B}}=1.5$ and $M_{1 \mathrm{~A}}=M_{1 \mathrm{~B}}=-1.5$ at zero temperature as shown in figure $2 \mathrm{c}$, and as the temperature increases they decrease and at the $T_{\mathrm{t}}, M_{1 \mathrm{~A}}=M_{1 \mathrm{~B}}$ jump discontinuously from their negative values to the positive values $\left(M_{2 \mathrm{~A}}=M_{2 \mathrm{~B}}\right.$ do exactly the opposite). The response functions also present jump discontinuities at the $T_{\mathrm{t}}$. However, in figure $2 \mathrm{~d}$, instead of $T_{\mathrm{t}}$ we have observed $T_{\mathrm{N}}$ for the given system parameters. $M_{2 \mathrm{~A}}=M_{2 \mathrm{~B}}=1.5$ and $M_{1 \mathrm{~A}}=M_{1 \mathrm{~B}}=-1.5$ at $k T / J_{1}=0.0$, but as $k T / J_{1}$ increases they decrease and combine at the $T_{\mathrm{N}}$ where the response functions again present peaks. The next figure is for the mixed phase $\mathrm{V}$ and is shown in figure $2 \mathrm{e}$. At $k T / J_{1}=0.0$, $M_{2 \mathrm{~B}}=-M_{2 \mathrm{~A}}=1.5$ and $M_{1 \mathrm{~B}}=-M_{1 \mathrm{~A}}=1.5$, as $k T / J_{1}$ increases they decrease again and at the 


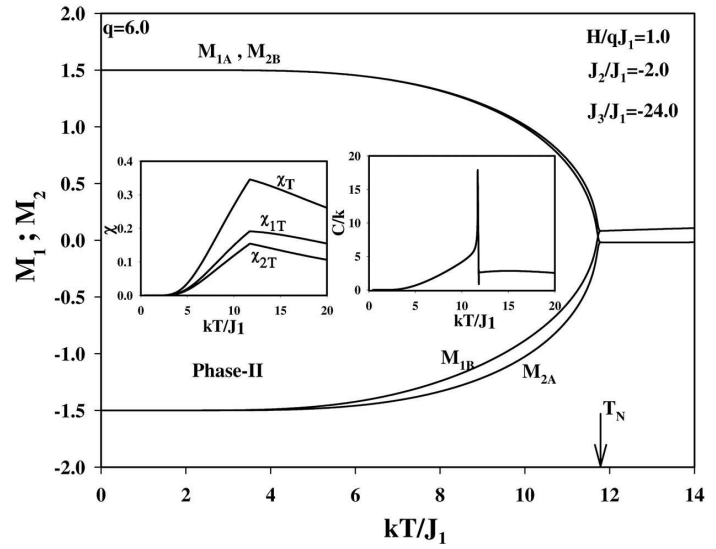

(a)

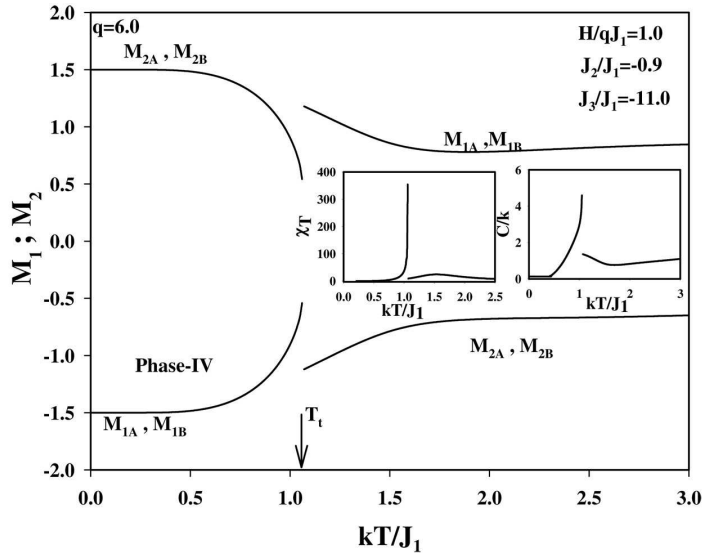

(c)

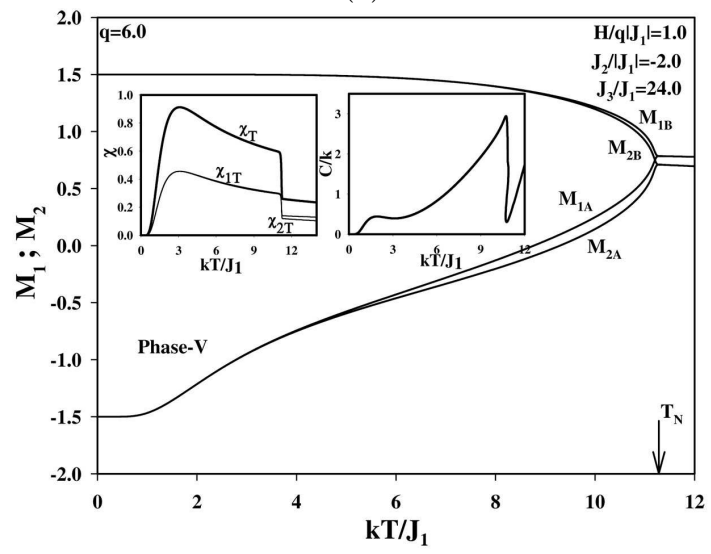

(e)

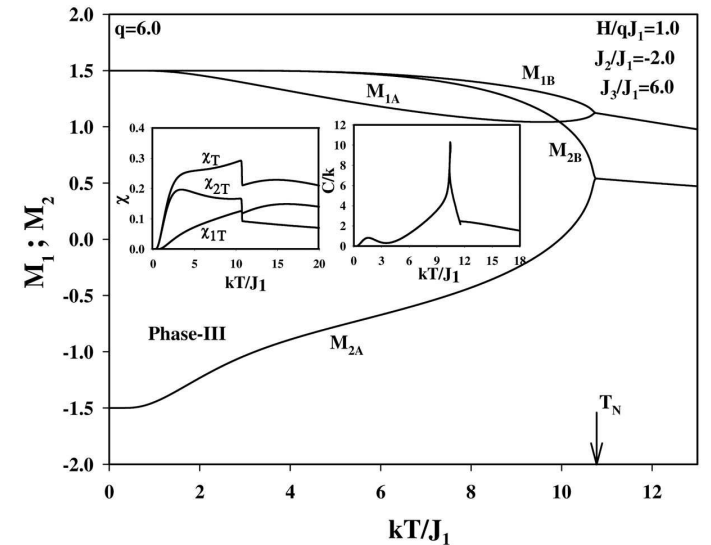

(b)

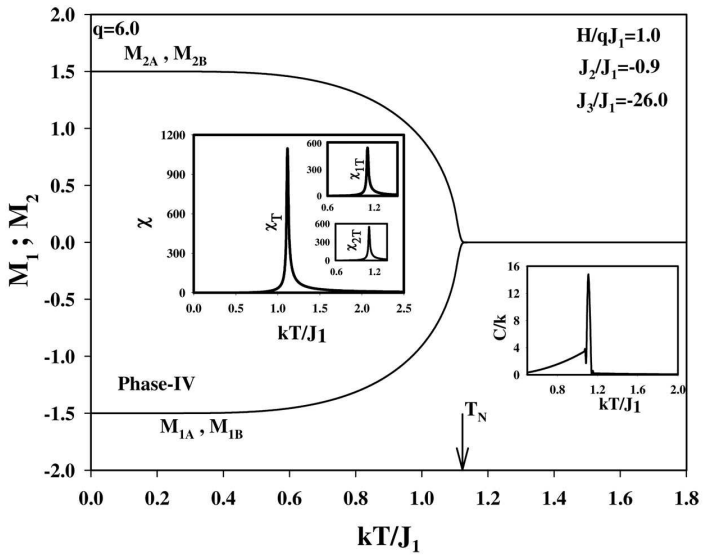

(d)

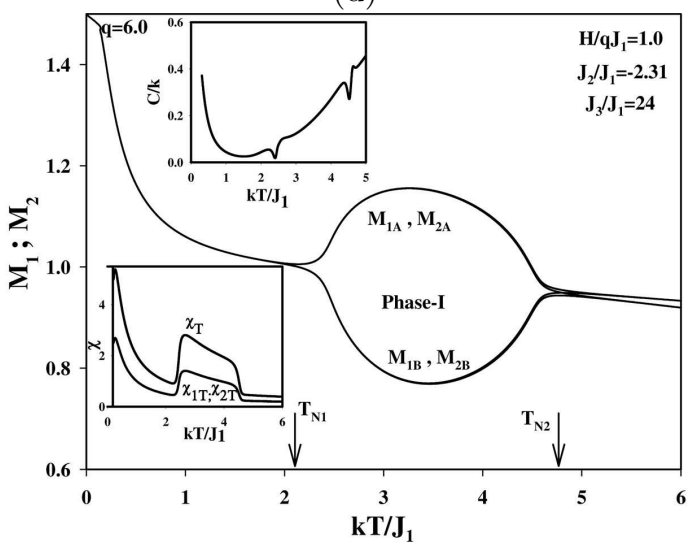

(f)

Figure 2. Thermal variations of the order-parameters and response functions for $q=6.0$ and $H / q J_{1}=1.0$; (a) $J_{3} / J_{1}=-24.0$ and $J_{2} / J_{1}=-2.0$, (b) $J_{3} / J_{1}=6.0$ and $J_{2} / J_{1}=-2.0$, (c) $J_{3} / J_{1}=-11.0$ and $J_{2} / J_{1}=-0.9$, (d) $J_{3} / J_{1}=-26.0$ and $J_{2} / J_{1}=-0.9$, (e) $J_{3} / J_{1}=24.0$ and $J_{2} / J_{1}=-2.0$, and (f) $J_{3} / J_{1}=24.0$ and $J_{2} / J_{1}=-2.31$. 
$T_{\mathrm{N}}, M_{1 \mathrm{~A}}=M_{1 \mathrm{~B}}$ and $M_{2 \mathrm{~A}}=M_{2 \mathrm{~B}}$ at different values of magnetizations. The response functions again show their peaks at the $T_{\mathrm{N}}$. Phase $\mathrm{I}$ is the FM phase and no phase transitions are expected when there are external magnetic fields acting on the layers which is well-known. On the contrary, in a very small transition region from AFM to FM phases two $T_{\mathrm{N}}$ 's are observed as shown in figure $2 \mathrm{f}$ (this will be clear when we present the phase diagrams). As seen $M_{1 \mathrm{~A}}=M_{1 \mathrm{~B}}$ and $M_{2 \mathrm{~A}}=M_{2 \mathrm{~B}}$ at zero temperature and they go together respectively until the first $T_{\mathrm{N} 1}$ at which they are separated. The separation of the sublattice magnetizations for each layer continue until a higher temperature $T_{\mathrm{N} 2}$ is reached, with $T_{\mathrm{N} 1}<T_{\mathrm{N} 2}$, where they combine again. This behavior is only seen for $q=4$ and 6 and which is the cause of the reentrant behavior of the $T_{\mathrm{N}}$-lines in the phase diagrams. The response functions present two peaks at these temperatures $T_{\mathrm{N} 1}$ and $T_{\mathrm{N} 2}$. Even if we did not present, we have also obtained two $T_{\mathrm{N}}$ 's in small transition regions from AFM phases to FM phase IV as in figure $2 \mathrm{f}$.

In conclusion we have illustrated the thermal changes of the order-parameters and the response functions and explained how they are used to determine the nature of the phase transitions. Now we are ready to give all possible distinct types of the temperature dependent phase diagrams for $q=3,4$ and 6 in the next section.

\section{The temperature dependent phase diagrams}

In the light of the previous two sections, we now give some of the distinct phase diagrams of the model. In the phase diagrams the $T_{\mathrm{N}}$-lines, the lines of the second-order phase transition temperatures, are indicated with dotted, dashed and solid lines for $q=3,4$ and 6 , respectively. Similarly, the arrows indicate the phase separation points according to the GS phase diagrams. The $T_{\mathrm{t}}$-lines are shown with chain lines. The temperature dependent phase diagrams are obtained on the $\left(J_{3} / J_{1}, k T / J_{1}\right),\left(J_{2} / J_{1}, k T / J_{1}\right),\left(H / J_{1}, k T / J_{1}\right),\left(J_{3} /\left|J_{2}\right|, k T /\left|J_{2}\right|\right),\left(J_{1} /\left|J_{2}\right|, k T /\left|J_{2}\right|\right)$ and $\left(H /\left|J_{2}\right|, k T /\left|J_{2}\right|\right)$ planes and we refer to them as the types A, B, C, D, E and F. The possible phase transitions involve the phases (V,I), (III,I), (II,IV), (III,IV), (V,III), (II,III) (II,III,V), (II,III,I), (IV,III,I) and (V,III,I) and which are labeled with $1,2, \ldots, 9,10$, respectively, for easiness. For example A1 is understood as the phase diagram on the $\left(J_{3} / J_{1}, k T / J_{1}\right)$ plane involving the phases $(\mathrm{V}, \mathrm{I})$ etc... The constant values of the system parameters that they are obtained for are indicated in the figures.

The first four phase diagrams are obtained for $H / q J_{1}=1.0$ according to the GS phase diagram figure 1a. Figure $3 \mathrm{a}$ is type A7 phase diagram. The $T_{\mathrm{N}}$-lines separate the ordered phases from the paramagnetic $(\mathrm{P})$ phase. As seen, they are constant at higher negative values of $J_{3} / J_{1}$ and which are at higher temperatures for higher $q$. As $J_{3} / J_{1}$ increases the $T_{\mathrm{N}}$ 's decrease and $T_{\mathrm{N}}$-lines present concavities for all $q$ about $J_{3} / J_{1}=0.0$. As $J_{3} / J_{1}$ increases further they become constant at some temperatures again. However, the $T_{\mathrm{N}}$ 's of the left wings are higher than the right wings. Since $J_{2}<0$ and $J_{3}<0$ favor the AFM phase II but while $J_{2}<0$ favor AFM phase, $J_{3}>0$ favor FM phase, so phase II is more resistive to temperature than the phase $\mathrm{V}$. The $T_{\mathrm{N}}$-lines do not terminate since both phases II and V are AFM type. The next one is presented in figure $3 \mathrm{~b}$ and it is A8 type. Again the $T_{\mathrm{N}}$-lines have constant temperatures in the phase region II at higher negative values of $J_{3} / J_{1}$, but as $J_{3} / J_{1}$ increases their temperatures increase continuously in the phase region III. As $J_{3} / J_{1}$ is increased further the $T_{\mathrm{N}}$-lines go to zero sharply in passing from phase III to phase I. This happens at lower $J_{3} / J_{1}$ for lower $q$. The $T_{\mathrm{N}}$-lines present a reentrant behavior for $q=4$ and 6 , due to the existence of two $T_{\mathrm{N}}$ 's (see figure $2 \mathrm{f}$ ). The transitions take place smoothly, i. e., the $T_{\mathrm{N}}$-lines start from AFM phase II, then go to the surface FM phase III and then pass FM phase I with a little reentrant part and finally terminate in the $\mathrm{P}$ phase. Figure $3 \mathrm{c}$ consists of three phase diagrams, i. e. while the main figure is for $J_{3} / q J_{1}=4.0$ and type B1, the insets $(i)$ and $(i i)$ are for $J_{3} / q J_{1}=1.0$ and -4.0 , and types B2 and B3, respectively. They are very similar to each other, that is, they start from higher temperatures at higher negative values of $J_{2} / J_{1}$ for higher q, and as $J_{2} / J_{1}$ increases their $T_{\mathrm{N}}$ 's decrease linearly and close to zero temperature some of them make little curves and then they all go to zero temperature at the same value of $J_{2} / J_{1}$. The reentrant behavior is seen for $q=6$ in B1 and for $q=4$ and 6 in B2, but none was seen in B3. The final figure obtained 


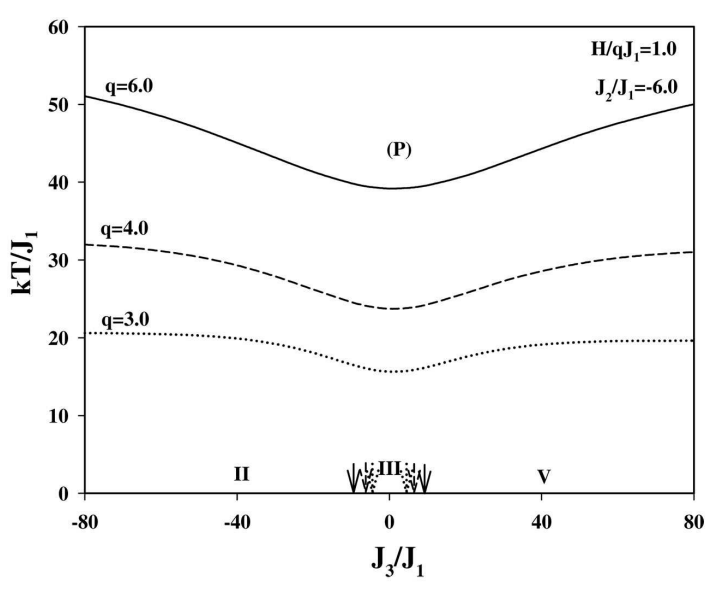

(a)

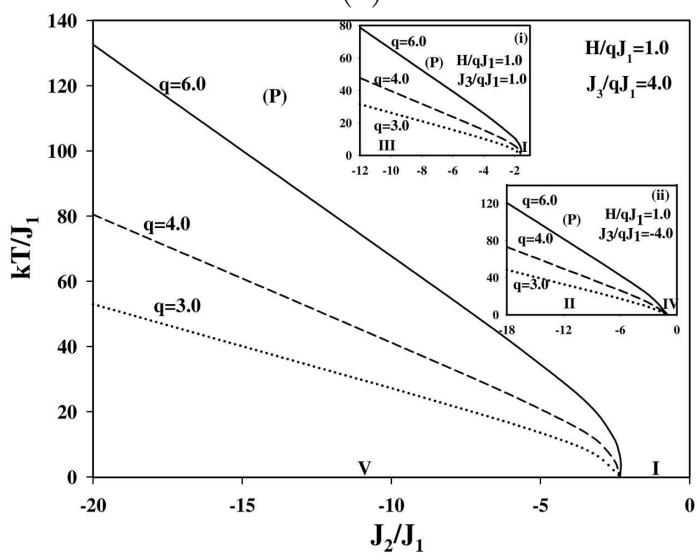

(c)

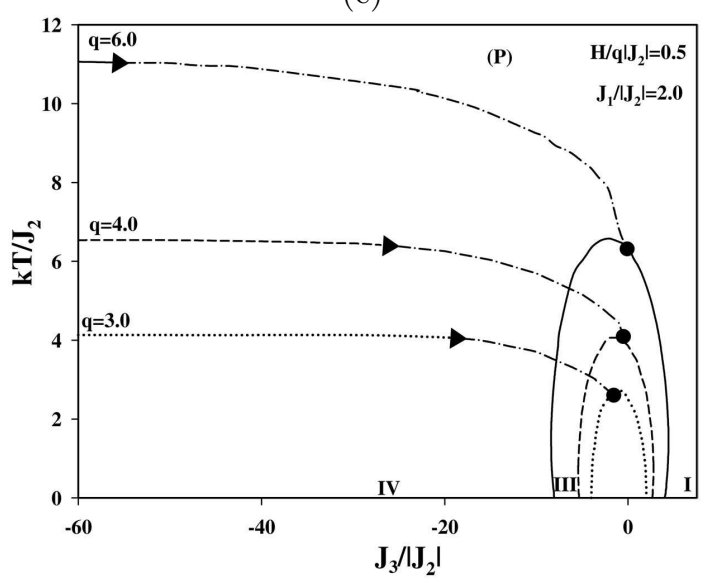

(e)

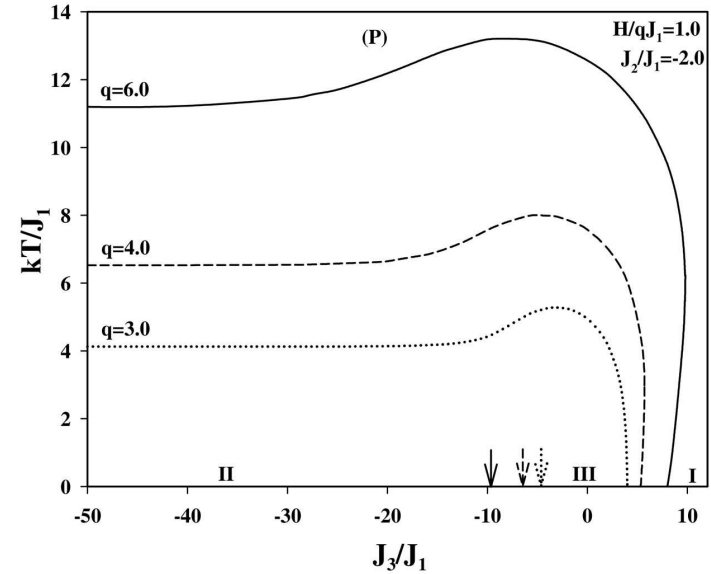

(b)

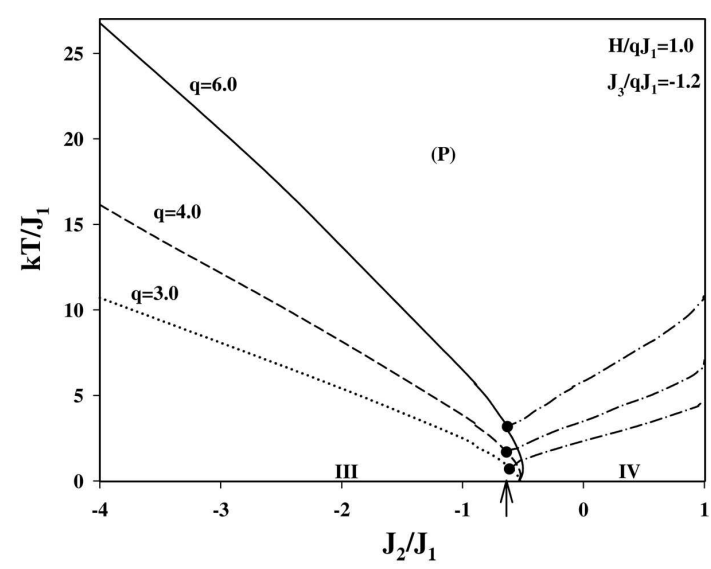

(d)

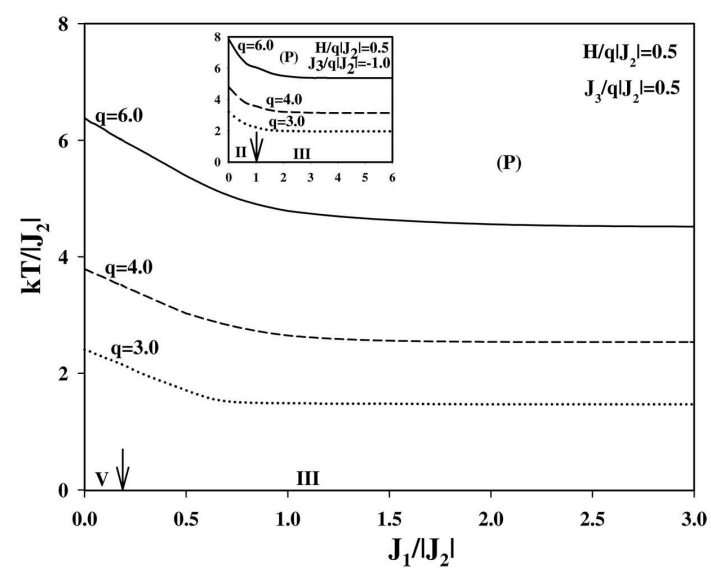

(f)

Figure 3. Some of the distinct phase diagrams of the model. In the guidance of figure 1a; (a)type A7, (b) type A8, (c) types B1, B2 and B3 and (d) type B4, in accord with figure 1b; (e) type D9 and (f) types E5 and E6, by the use of figure 1c. 


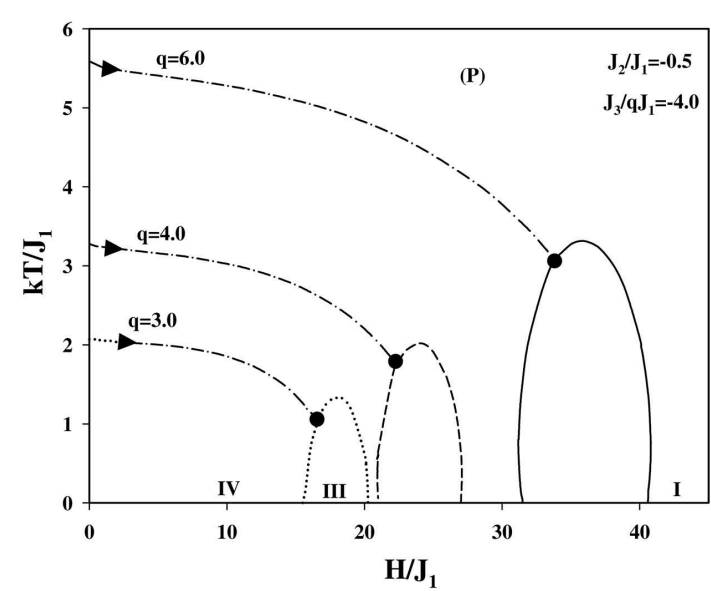

$(\mathrm{g})$

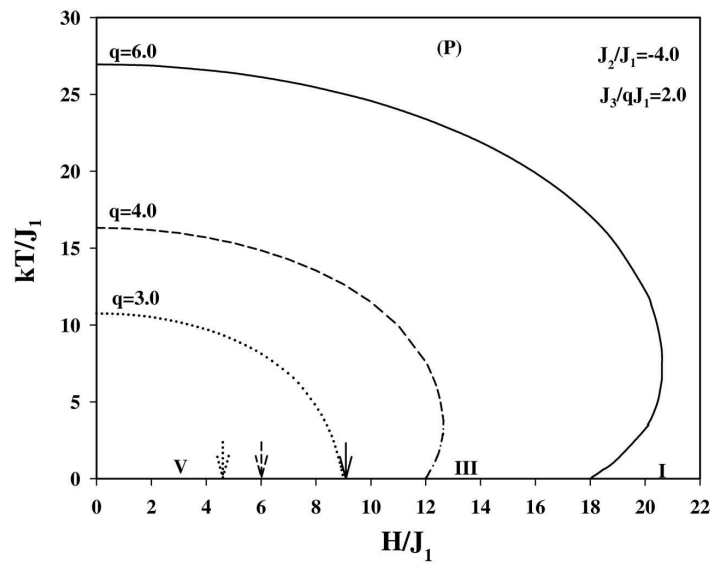

(h)

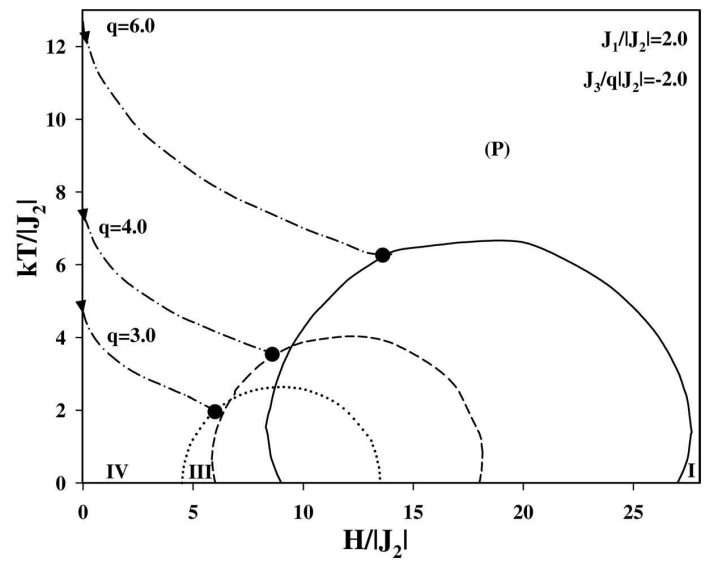

(i)

Figure 3. Continuation: (g) type C9 and (h) type C10 and the last one with the help of figure 1d; (i) type F9.

from figure 1a is B4 type and is presented in figure $3 \mathrm{~d}$. The behaviors of the $T_{\mathrm{N}}$-lines are similar to the previous figure. But now we also see the existence of the $T_{\mathrm{t}}$-lines (see figure $2 \mathrm{c}$ ) which emerge from the $T_{\mathrm{N}}$-lines in phase region IV and increases with increasing $J_{2} / J_{1}$. Thus, the connection points of these lines are the critical end points. The reentrant behavior is again observed for $q=4$ and 6 .

Figure $1 \mathrm{~b}$ is used in our upcoming three phase diagrams. The phase diagram of type D9 is one of the most interesting and is illustrated in figure 3e. Phase IV is FM phase and we do not expect any $T_{\mathrm{N}}$ 's when the layers are linked with $H$, but due to the competition between $J_{3}$ and $H$ for $J_{1} /\left|J_{2}\right|=2.0$ we actually observe $T_{\mathrm{N}}$ 's (see figure $2 \mathrm{f}$ ). Thus, $T_{\mathrm{N}}$-lines start from higher constant temperatures for higher $q$ at higher negative values of $J_{3} /\left|J_{2}\right|$. As $J_{3} /\left|J_{2}\right|$ increases the $T_{\mathrm{N}}$-lines become the $T_{\mathrm{t}}$-lines (see figure $2 \mathrm{c}$ ). Thus, the connection points of these lines are the tricritical points. The temperatures of these $T_{\mathrm{t}}$-lines decrease up to the critical end points where they combine with the corresponding $T_{\mathrm{N}}$-lines for each $q$ in the shape of half upward ellipsoid for each $q$. The combinations of the $T_{\mathrm{N}^{-}}$and $T_{\mathrm{t}}$-lines are actually unstable since their free energies are higher in comparison with the half ellipsoidal ones. Note also that the $T_{\mathrm{N}}$-lines in the shape of half upward ellipsoids bind the phase III for each $q$. The reentrant behavior is also obvious in both of the legs of $T_{\mathrm{N}}$-lines for $q=4$ and 6 . The next phase diagram is to illustrate E5 and E6 types of phase transitions as seen in figure 3f. They are obtained for $J_{3} / q\left|J_{2}\right|=0.5$ and -1.0 (see inset), respectively, and are very similar to each other. The $T_{\mathrm{N}}$-lines start from higher temperatures for 
higher $q$ at $J_{1} /\left|J_{2}\right|=0.0$, and as it increases their $T_{\mathrm{N}}$ 's decrease and become constant at lower temperatures for higher $J_{1} /\left|J_{2}\right|$. The $T_{\mathrm{N}}$-lines always exist and separate the ordered phases from $\mathrm{P}$ phase.

Figures $3 \mathrm{~g}$ and $3 \mathrm{~h}$ are obtained according to the GS phase diagram figure 1c. The first one is C9 type, obtained for $J_{3} / q J_{1}=-4.0$ and where all possible critical phenomena of this work were displayed as in figure 3e. Very short $T_{\mathrm{N}}$-lines are seen for very low values of $H / J_{1}$. It is well-understood that the second-order transitions are expected at zero external magnetic field for the phase IV (FM phase), which is indeed the case. These $T_{\mathrm{N}}$-lines end and therefrom the $T_{\mathrm{t}}$-lines emerge for all $q$. Thus, tricritical points are observed. These $T_{\mathrm{t}}$-lines end at critical end points on the half upward ellipsoidal $T_{\mathrm{N}}$-lines as in figure $3 \mathrm{e}$. The unstable part of the critical lines, i. e. the combinations of the $T_{\mathrm{t}}$ - and $T_{\mathrm{N}}$-lines, separate the $\mathrm{P}$ phase from phase IV. The ellipsoid shaped $T_{\mathrm{N}}$-lines are lined up from lower to higher $H / J_{1}$ values with increasing $q$. Again the reentrant behavior is seen in both legs of these $T_{\mathrm{N}}$-lines for $q=4$ and 6 . The last one of this group is C10 type as seen in figure $3 \mathrm{~h}$. As usual the $T_{\mathrm{N}}$-lines start from higher temperatures for zero $H / J_{1}$ and as $H / J_{1}$ increases their $T_{\mathrm{N}}$ 's decrease and they go to zero temperatures at lower $H / J_{1}$ for lower $q$. It is now quite obvious that the reentrant behavior exists for $q=4$ and 6 again.

The last phase diagram of this work is obtained by using the GS phase diagram figure 1d and it is F9 type as shown in figure 3i. In this phase diagram in comparison with the type C9, the roles of $J_{1}$ and $J_{2}$ are exchanged. Again a few $T_{\mathrm{N}}$ 's exist at zero and at about zero $H /\left|J_{2}\right|$ and so they make very small $T_{\mathrm{N}}$-lines for each $q$. From the ends of these $T_{\mathrm{N}}$-lines for each $q$, the $T_{\mathrm{t}}$-lines emerge. Thus, the tricritical points exist. The temperatures of these $T_{\mathrm{t}}$-lines decrease with increasing $H /\left|J_{2}\right|$ and they combine with their corresponding closed $T_{\mathrm{N}^{-}}$lines at the critical end points. The closed $T_{\mathrm{N}^{-}}$ lines are much wider than our observed ellipsoidal ones. Again the reentrant behaviors are much clearer for $q=4$ and 6 . We should mention that we have obtained all possible phase diagrams of the model according to our classification scheme as explained in the first paragraph of this section, but we gave only some of the distinct types of the phase diagrams.

\section{Summary and conclusions}

In summary, we have considered spin-3/2 Ising model on two symmetrically placed Bethe lattices with the upper one having only FM and the lower one having only AFM interactions, each with a branching ratio of $q$ Ising spins, and each are coupled via either FM or AFM interactions. The model is examined by using the recursion relations in a pairwise approach for the given coordination numbers $q=3,4$ and 6 with equal external magnetic fields linked to the layers. The phase diagrams are classified and obtained on different planes for the given system parameters by studying in detail the ground state (GS) phase diagrams and the thermal variations of the order parameters and the response functions, i. e. the susceptibility and the specific heat. We have found that the model presents both second- and first-order phase transitions, and where their lines are combined is the tricritical point. The critical end points, two $T_{\mathrm{N}}$-lines emerging from the end of the $T_{\mathrm{t}}$-lines, are also found to exist. The reentrant behavior is seen when the model presents two Néel temperatures for $q=4$ and 6 only.

Now we present a brief discussion of what we have found: $(i)$ The same GS configurations are found with those of the FM/FM interactions for spin-3/2 model [8], but with different GS phase diagrams. (ii) The first-order phase transitions are always seen for transitions from phase IV, i. e. compensated phase, to $\mathrm{P}$ phase, the paramagnetic phase, as shown in figures $3 \mathrm{~d}$, e, g and i. (iii) The tricritical points and critical end points are found here in contrast to the case with FM/FM interactions [8]. (iv) The reentrant behavior is only exhibited for transitions from phase III to phase I or IV for both $q=4$ and 6 and is only seen for $q=6$ for the phase transitions from phase $\mathrm{V}$ to phase I. The reentrant behavior was not observed for the FM/FM interactions with spin-3/2 model. Our final result is $(v)$ The critical temperatures are seen at higher temperatures for higher coordination numbers.

We can also give some comparisons, at least, for the cases with spin-1/2 [22] and spin-1 [23] FM/AFM models; $(i)$ With spin-1/2: No first-order phase transitions were exhibited, but the spin- 
$3 / 2$ model displays both the first- and second-order phase transitions. Therefore, the tricritical points and critical end points are also displayed. The reentrant behavior was only seen when $q=6$ in the spin- $1 / 2$ case, but it was also seen for $q=4$ in the spin- $3 / 2$ case. (ii) With spin- 1 : The first-order phase transitions are always exhibited for the transitions from phase IV to phase $\mathrm{P}$ for spin-3/2 model but they were not always exhibited for the spin-1 model. This is the only difference in comparison with the spin-1 case, so it is much less than our expectations. As a last word, the critical temperatures are seen at higher temperatures for higher spin values as expected.

Since this is the first theoretical study of this kind for spin-3/2 FM/AFM interactions on a two-layer lattice, we hope that other researchers will also get interested in considering the model using other techniques.

\section{Acknowledgement}

This work is supported by The Scientific and Technological Research Council of Turkey (TUBITAK) with Fund No. 107T358.

\section{References}

1. Sivardière J., Blume M., Phys. Rev. B, 1972, 5, 1126.

2. Krinsky S., Mukamel D., Phys. Rev. B, 1975, 11, 399.

3. Baibich M.N., Broto J.M., Fert A., Nguyen Van Dau F., Petroff F., Eitenne P., Creuzet G., Friederich A., Chazelas J., Phys. Rev. Lett., 1988, 61, 2472; Binasch G., Grunberg P., Saurenbach F., Zinn W., Phys. Rev. B, 1989, 39, 4828.

4. Sayama J., Asahi T., Mizutani K., Osaka T., J. Phys. D: Appl. Phys., 2004, 37, L1-L4.

5. Wu R., Freeman A.J., Phys. Rev. B, 1992, 45, 7205; Donath M., J. Phys.: Condens. Matter 11, 1999, 9421.

6. O'Handley R.C., Sun S.W., Phys. Rev. Lett., 1991, 66, 2798; Bochi G., Song O., O'Handley R.C., Phys. Rev. B, 1994, 50, 2043.

7. Horiguchi T., Lipowski A., Tsushima N., Physica A, 1996, 224, 626.

8. Albayrak E., Yilmaz S., Akkaya S., J. Magn. Magn. Mater, 2007, 310, 98.

9. Jaščur M., Kaneyoshi T., Physica A, 1995, 220, 542.

10. Nogues J., Lederman D., Moran T.J., Schuller I.K., Phys. Rev. Lett., 1996, 76, 4624.

11. Sort J., Suriñach S., Muñoz J.S., Baró M.D., Nogués J., Chouteau G., Skumryev V., Hadjipanayis G.C., Phys. Rev. B, 2002, 65, 174420.

12. Malkinski L., O'Keevan T., Camley R.E., Celinski Z., He J., Zhou W.L., Hecker M., Schneider C.M., Szade J., Skrzypek D., J. Vac. Sci. Technol. A, 2003, 21(4), 1162.

13. Mushailov E.S., Vasiliev B.V., J. Magn. Magn. Mater., 2004, 279, 235.

14. Yi Y.B., Ding J., J. Magn. Magn. Mater., 2006, 303, e160.

15. Yamada T.K., Martinez E., Vega A., Robles R., Stoeffler D., Vázquez de Parga A.L., Mizoguchi T., van Kempen H., Nanotechnology, 2007, 18, 235702.

16. Wang J., Kuch W., Chelaru L.I., Offi F., Kotsugi M., Kirschner J., J. Phys.: Condens. Matter, 2004, 16, 9181.

17. Park C.M., Lee K.A., Hwang D.G., Lee S.S., Kim M.Y., Rhee J.R., J. of Korean Phys. Soc., 1997, 31, 508.

18. Camarero J., Sort J., Hoffmann A., García-Martin J.M., Dieny B., Miranda R., Nogués J., Phys. Rev. Lett., 2005, 95, 057204.

19. Hu C.-K., Izmailian N.Sh., Oganesyan K.B., Phys. Rev. E, 1999, 59, 6489.

20. Lyra M.L., da Silva C.R., Phys. Rev. B, 1993, 47, 526.

21. Monroe J.L., Phys. Rev. E, 2005, 71, 017105.

22. Albayrak E., Phys. Stat. Sol. b, 2009, 246, 226.

23. Albayrak E., Akkaya S., Cengiz T., J. Magn. Magn. Mater., 2009, 321, 108; Albayrak E., Canko O., Physica A, 2007, 373, 363.

24. Canko O., Albayrak E., Phys. Rev. E, 2007, 75, 011116.

25. Kurota M., Kikuchi R., Watari T., J. Chem Phys., 1953, 21, 434; Domb C., Adv. Phys., 1960, 9, 208.

26. Hu C.-K., Izmailian N.Sh., Phys. Rev. E, 1998, 58, 1644; Izmailian N.Sh., Hu C.-K., Physica A, 1998, 254, 198. 
27. Tucker J.W., J. Magn. Magn. Mater., 1999, 195, 733; Albayrak E., Keskin M., J. Magn. Magn. Mater., 2003, 261, 196; Albayrak E., Yigit A., Physica A, 2005, 349, 471; Albayrak E., Yigit A., Phys. Stat. Sol. b, 2005, 242, 1510 .

28. Albayrak E., Yilmaz S., Akkaya S., Physica A, 2007, 381, 189.

\title{
Модель Ізинга зі спіном 3/2 на двошаровій гратці Бете зі взаємодіями феромагнетик-антиферомагнетик
}

\author{
Е.Альбайрак, А.Їгіт \\ Фізичний факультет, Університет Ерсієс, 38039 Кайсері, Туреччина \\ Отримано 2 лютого 2009 р., в остаточному вигляді - 25 травня 2009 р.
}

Двошарова гратка Бете з вузлами, зайнятими атомами зі спіном 3/2, є точно розв'язаною, використовуючи рекурентні співвідношення у парному підході з однаковими магнітними полями, що діють на шари. Припускається, що спіни верхнього і нижнього шарів мають відповідно, феромагнітні (ФМ) та антиферомагнітні (АФМ) взаємодії, а взаємодія між сусідніми спінами шарів є ФМ або АФМ типу. Вивчаються фазові діаграми моделі на різних площинах для даного набору системних параметрів шляхом отримання основного стану фазової діаграми і температурних змін параметрів порядку та функцій відгуку, зокрема, сприйнятливості і питомої теплоємності. Показано, що модель демонструє фазові переходи як другого, так першого роду. Видно реверсивну поведінку, коли модель демонструє дві температури Нееля для вищих значень $q$. Також отримано трикритичну точку і критичну кінцеву точку.

Ключові слова: гратка Бете, спін, основний стан, ФМ/АФМ, шари, фаза

PACS: $05.50 .+q, 68.35 . R h, 64.60 . C n$ 
